\title{
An algorithmic theory of learning: Robust concepts and random projection
}

\author{
Rosa I. Arriaga · Santosh Vempala
}

Received: 11 August 2003 / Revised: 27 July 2004 / Accepted: 10 November 2005 / Published online: 28 March 2006

Springer Science + Business Media, LLC 2006

\begin{abstract}
We study the phenomenon of cognitive learning from an algorithmic standpoint. How does the brain effectively learn concepts from a small number of examples despite the fact that each example contains a huge amount of information? We provide a novel algorithmic analysis via a model of robust concept learning (closely related to "margin classifiers"), and show that a relatively small number of examples are sufficient to learn rich concept classes. The new algorithms have several advantages - they are faster, conceptually simpler, and resistant to low levels of noise. For example, a robust half-space can be learned in linear time using only a constant number of training examples, regardless of the number of attributes. A general (algorithmic) consequence of the model, that "more robust concepts are easier to learn", is supported by a multitude of psychological studies.
\end{abstract}

Keywords Learning $\cdot$ Cognition $\cdot$ Random projection $\cdot$ Robust concepts

\section{Introduction}

One motivation of computational learning theory is to gather insight into cognitive processes. The exact physical processes underlying learning, indeed any aspect of cognition, are far from being understood. Even from a purely theoretical standpoint, it is mostly a mystery as to how the brain copes with huge amounts of data. How does the brain effectively learn concepts

\section{Editor: Shai Ben-David}

A preliminary version of this paper appeared in the Proc. of the Symposium on the Foundations of Computer Science, 1999

R. I. Arriaga

Department of Psychology, Southern New Hampshire University

e-mail: r.arriaga@snhu.edu

S.Vempala $(\bowtie)$

Department of Mathematics, M.I.T.

e-mail: vempala@math.mit.edu 
from a relatively small number of examples, when each example consists of a huge amount of information?

There are at least two approaches to explaining this phenomenon. The first, due to Valiant, is attribute-efficient learning (Valiant, 1998; Littlestone, 1987, 1991). In this model, it is assumed that the target concept is simple in a specific manner: it is a function of only a small subset of the set of attributes, called the relevant attributes, while the rest are irrelevant. From this assumption one can typically argue that the VC-dimension of the resulting concept class is a function of only the number of relevant attributes $(k)$, and hence derive a bound on the number of examples required. Unfortunately, although the model is theoretically clean and appealing, it is not known how to learn anything more complex than a disjunction of variables (without membership queries). Further, it is NP-hard to learn a disjunction of $k$ variables as a disjunction of fewer than $k \log n$ variables (where $n$ is the total number of variables).

In this paper, we study a different approach based on a simple idea which is illustrated in the following example. Imagine a child learning the concept of an "elephant". We point the child to pictures of elephants or to real elephants a few times and say "elephant", and perhaps to a few examples of other animals and say their names (i.e., "not elephant"). From then on, the child will almost surely correctly label only elephants as elephants. On the other hand, imagine a child learning the concept of "African elephant" (as opposed to the Indian elephant) just from examples. It will probably take many more examples, and perhaps even be necessary to explicitly point out the bigger ears of the African elephant.

The crucial difference in the two concepts above is not in the number of attributes, or even in the number of relevant attributes of the examples presented, but in the similarity of examples with the same label and in the dissimilarity of examples with different labels. There is a clearer demarcation between elephants and non-elephants than there is between African elephants and Indian elephants. This notion will be formalized later as the robustness of a concept. An alternative perspective of robustness is that it is a measure of how much the attributes of an example can be altered without affecting the concept. The main feature of robust concepts is that the number of examples and the time required to learn a robust concept can be bounded as a function of the robustness (denoted by a parameter $\ell$ ), and do not depend on the total number of attributes. The model and the parameter $\ell$ are defined precisely in Section 2. As we discuss there, the model is very closely related to Large Margin classifiers studied in machine learning, that are in turn the basis for Support Vector Machines (Vapnik, 1995; Cortes \& Vapnik, 1995).

In the robust concept model, the main new observation is that we can employ a general procedure to reduce the dimensionality of examples, independent of the concept class. While reducing the dimensionality of examples, we would like to preserve concepts. So, for example, if our original concept class is the set of half-spaces (linear thresholds) in $n$-dimensional space, we would like to map examples to a $k$-dimensional space, where $k$ is much smaller than $n$, and maintain the property that some half-space in the $k$-dimensional space correctly classifies (most of) the examples. We show that Random Projection, the technique of projecting a set of points to a randomly chosen low-dimensional space, is suitable for this purpose. It has been observed that random projection (approximately) preserves key properties of a set of points, e.g., the distances between pairs of points (Johnson \& Lindenstrauss, 1984); this has led to efficient algorithms in several other contexts (Kleinberg, 1997; Linial, et al., 1994; Vempala, 2004). In Section 3, we develop "neuronal" versions of random projection, i.e., we demonstrate that it is easy to implement it using a single layer of perceptrons where the weights of the network are chosen independently and from any one of a class of distributions; this class includes discrete distributions such as the picking 1 or -1 with equal probability. Our theorems 
here can be viewed as extensions/refinements of the work of Johnson \& Lindenstrauss (1984) and Frankl and Maehara (1988).

Then we address the question of how many examples are needed to efficiently learn a concept with robustness $\ell$. We begin with the concept class of half-spaces with $n$ attributes. In this case, it is already known that one needs $O\left(1 / \ell^{2}\right)$ examples (Bartlett \& Shawe-Taylor, 1998; Vapnik, 1995; Freund \& Schapire, 1999). Here we show that a simple algorithm based on random projection gives an alternative proof of such a guarantee.

Next we consider other rich concept classes, namely intersections of half-spaces and ellipsoids. Using neuronal random projection, we demonstrate that the examples can first be projected down to a space whose dimension is a function of $\ell$, and in some cases an additional parameter of the concept class (e.g. the number of half-spaces when the concept class is intersections of half-spaces etc.), but does not depend on the number of attributes of the examples. This then allows us to bound the number of examples required to learn the concepts as a function of $\ell$, independent of the original number of attributes, via well-known generalization theorems based on the VC-dimension (Vapnik \& Chervonenkis, 1971).

The proposed algorithms are fast-their running time is linear in $n$ - since after random projection (which takes time linear in $n$ ), all the work happens in the smaller-dimensional space with a small number of sample points. Indeed, this suggests that the algorithms studied here could be used in SVM's in place of current solutions (Cortes \& Vapnik, 1995; Freund \& Schapire, 1999) such as quadratic optimization in a dual space called the kernel space.

In Section 4.4, we mention the noise tolerance properties of the algorithms, notably that agnostic learning is possible, and (equivalently) that it is possible to find hypotheses that minimize the number of misclassified points, for fairly low robustness.

\subsection{Related work}

The main contribution of this paper is a new perspective on learning via a connection to dimension reduction. This facilitates efficient algorithms which use small sample sizes. It also gives a simple intuitive way to see the $O\left(1 / \epsilon^{2}\right)$ sample complexity bounds of margin classifiers (SVM's) (Bartlett \& Shawe-Taylor, 1998). It is related to previous work (Schapire et al., 1998) which showed that generalization error can be bounded in terms of the observed margin of examples (a more refined notion of margin is used there, but is similar in spirit). As we discuss in Section 5.1, it seems to fit well with attempts to model cognition on a computational basis (Valiant, 1998), and predicts the commonly observed phenomenon that finer distinctions take more examples. From a purely computational viewpoint, these are simple new algorithms for fundamental learning theory problems, that might be practical.

There have been further applications of random projection in learning theory subsequent to this work. Garg, et al. (2002) and Garg and Roth (2003) have pursued similar ideas, developing the related notion of projection profile. Recently, Balcan, et al. (2004) have used random projection to give an efficient new interpretation of kernel functions. Klivans and Servedio (2004) have used polynomial threshold functions in the context of robust concepts to get substantially improved time bounds. Specifically, they give faster algorithms for learning intersections (and other functions) of $t$ half-spaces (with some increase in the sample complexity). Finally, Ben-David, et al. (2002) have used random projection to show an interesting lower bound on learning with half-spaces. They prove that "most" concept classes of even constant VC-dimension cannot be embedded into half-spaces where the dimension of the Euclidean space is small or the margin is large. Thus, algorithms based on first transforming to half-spaces cannot gain much in terms of the margin or the dimension. 


\section{The model}

To describe the model, we adopt the terminology used in the literature. We assume that attributes are real valued; an example is a point in $\boldsymbol{R}^{n}$; a concept is a subset of $\boldsymbol{R}^{n}$. An example that belongs to a concept is labelled positive for the concept, and an example that lies outside the concept is labelled a negative example.

Given a set of labelled examples drawn from an unknown distribution $\mathcal{D}$ in $\boldsymbol{R}^{n}$, and labelled according to an unknown target concept the learning task is to find a hypothesis with low error. A hypothesis is a polynomial-time computable function. The error of a hypothesis $h$ with respect to the target concept is the probability that $h$ disagrees with the target function on a random example drawn from $\mathcal{D}$. Thus, if $h$ has error $\epsilon$, then the probability for a random $x$ that $h(x)$ disagrees with the target concept is at most $\epsilon$. So, given an error parameter $\epsilon$ and a confidence parameter $\delta$, with probability at least $1-\delta$, the algorithm has to find a concept that has error at most $\epsilon$ on $\mathcal{D}$ (Valiant, 1984).

The basic insight of the new model is the idea of robustness (implicit in earlier work). Intuitively, a concept is "robust" if it is immune to attribute noise. That is, modifying the attributes of an example by some bounded amount does not change its label. Another interpretation is that points with different labels are far apart. This is formalized below:

Definition 1. For any real number $\ell>0$, a concept $C$ in conjunction with a distribution $\mathcal{D}$ in $\boldsymbol{R}^{n}$, is said to be $\ell$-robust, if

$$
\mathrm{P}_{\mathcal{D}}(x \mid \exists y: \operatorname{label}(x) \neq \operatorname{label}(y),\|x-y\| \leq \ell)=0
$$

The norm $\|x-y\|$ is the Euclidean distance between $x$ and $y$. This can be replaced by other norms, but we use the Euclidean norm in this paper. The probability is over all points $x$ with the property that there is some point $y$ with a different label within a distance $\ell$. In other words, a concept is $\ell$-robust if there is zero probability of points being within $\ell$ of the boundary of the concept. The definition could be weakened by requiring only that the above probability should be negligible (e.g. $1 / 2^{n}$ ). When $\mathcal{D}$ is over a discrete subset of $\boldsymbol{R}^{n}$, then this has a simple interpretation. A ball of radius $\ell$ around any point $x$ of non-zero probability lies entirely on one side of the concept, i.e., every point in the ball has the same label as $x$. To avoid scaling issues, we usually consider only distributions whose support is (a subset of) the unit ball in $\boldsymbol{R}^{n}$, i.e., all examples given to the algorithm will have length at most 1 (alternatively, one could incorporate normalize the distance between examples by their length, but we find our definition more convenient). Given access to examples from a robust concept, and parameters $\epsilon, \delta$, a learning algorithm succeeds and is said to $(\epsilon, \delta)$-learn if, with probability at least $1-\delta$, it produces a hypothesis that is consistent with at least $1-\epsilon$ of the example distribution. Note that strictly speaking this is not PAC-learning since robustness restricts the example distribution.

In what follows, we present tools and algorithms for learning robust concepts. It is worth noting that "robustness" refers only to the target concept; it is not required of all concepts in the class.

\subsection{Connection to existing models}

The model is closely related to large margin classifiers used in Support Vector Machines (Bartlett \& Shawe-Taylor, 1998). Indeed, for the concept class of half-spaces, the robustness 
as defined here is exactly the largest possible margin of a correctly classifying half-space (with the normalization that all the examples are from the unit ball). In general, however, there is a subtle but important difference. Whereas in SVM's the margin is measured in the "lifted" space where concepts have been transformed to half-spaces, in our model we measure robustness in the space in which examples are presented to us (and hence the natural relationship with attribute noise). The robustness is also closely related to the parameter $\gamma$ used in the definition of the fat-shattering dimension (Kearns \& Schapire, 1994; Bartlett \& Shawe-Taylor, 1998), and once again coincides (up to a scaling factor) in the case of half-spaces.

\section{The main tool: "neuron-friendly" random projection}

In this section we develop "neuronal" versions of random projection, including a discrete version, and provide probabilistic guarantees for them, all with transparent proofs. Besides being neuron-friendly, these versions of random projection are easier to implement.

To project a given point $u \in \boldsymbol{R}^{n}$ to a $k$-dimensional space, we first choose $k$ random vectors $R_{1}, \ldots, R_{k}$ (we will shortly discuss suitable probability distributions for these vectors). Then we compute a $k$-dimensional vector $u^{\prime}$ whose coordinates are the inner products $u_{1}^{\prime}=$ $R_{1}^{T} \cdot u, \ldots, u_{k}^{\prime}=R_{k}^{T} \cdot u$. If we let $R$ be the $n \times k$ matrix whose columns are the vectors $R_{1}, \ldots, R_{k}$, then the projection can be succinctly written as $u^{\prime}=R^{T} u$. To project a set of points $u^{1}, \ldots, u^{m}$ in $\boldsymbol{R}^{n}$ to $\boldsymbol{R}^{k}$, we choose a random matrix $R$ as above, and compute the vectors $R^{T} u^{1}, \ldots, R^{T} u^{m}$.

Given the matrix $R$, the above procedure is a simple computational task. It has been shown that if $R$ is a random orthonormal matrix, i.e., the columns of $R$ are random unit vectors and they are pairwise orthogonal, then the projection preserves all pairwise distances to within a factor of $(1+\epsilon)$ for a surprisingly small value of $k$ of about $\log n / \epsilon^{2}$ (Johnson $\&$ Lindenstrauss, 1984). The main observation of this section is to show that this is a rather robust phenomenon, in that the entries of $R$ can be chosen from any distribution with bounded moments. In particular it suffices to use random matrices with independent entries chosen from a distribution with bounded support. It is then an easy consequence that the task of random projection can be achieved by a simple 1-layer neural network, viz., $k$ perceptrons (which compute linear combinations of their inputs) each with one output and the same $n$ inputs. The weights of the neural network are assumed to be random and independent. This is illustrated in Fig. 1. Let $r \in \boldsymbol{R}^{n}$ be a random vector whose coordinates are independent and identically distributed. We highlight the following two possibilities for the distribution of the coordinates: (a) the standard normal distribution, with mean 0 and variance 1 , referred to as $N(0,1)$, (b) the discrete distribution defined by $r_{i}=1$ with probability $\frac{1}{2}$ and $r_{i}=-1$ with probability $\frac{1}{2}$, which we will refer to as $U(-1,1)$. Following the conference version of this paper (Arriaga \& Vempala, 1999), another proof for the case $U(-1,1)$ has also appeared (Achlioptas, 2001). The following well-known lemma will be useful. We provide a proof for convenience.

Lemma 1. Let $X$ be drawn from $N(0, \sigma)$, the normal distribution with mean zero and standard deviation $\sigma$. Then for any $\alpha<\frac{1}{2 \sigma^{2}}$,

$$
\mathrm{E}\left(e^{\alpha X^{2}}\right)=\frac{1}{\sqrt{1-2 \alpha \sigma^{2}}} .
$$


Fig. 1 Neuronal Random Projection

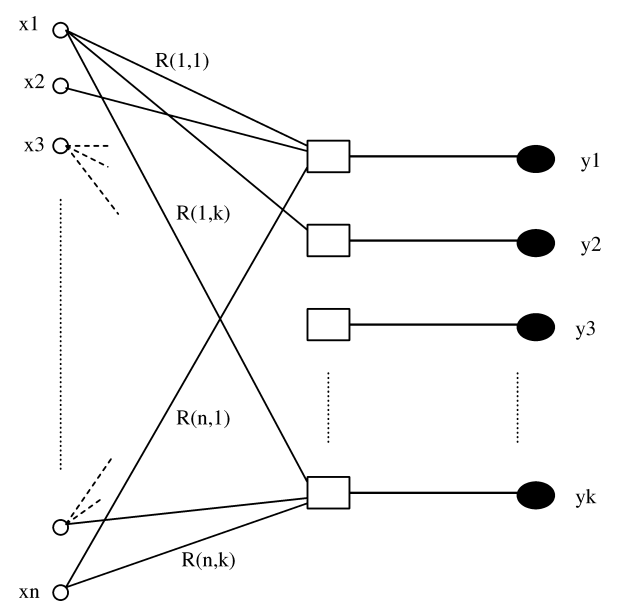

Proof: We recall the density function of $N(0, \sigma)$, the normal distribution with mean 0 and standard deviation $\sigma$, to be

$$
\frac{1}{\sqrt{2 \pi} \sigma} e^{-\frac{x^{2}}{2 \sigma^{2}}} .
$$

Using this,

$$
\begin{aligned}
\mathrm{E}\left(e^{\alpha X^{2}}\right) & =\int_{-\infty}^{\infty} e^{\alpha x^{2}} \frac{1}{\sqrt{2 \pi} \sigma} e^{-\frac{x^{2}}{2 \sigma^{2}}} d x \\
& =\int_{-\infty}^{\infty} \frac{1}{\sqrt{2 \pi} \sigma} e^{-\frac{x^{2}}{2 \sigma^{2}}\left(1-2 \alpha \sigma^{2}\right)} d x \\
& =\frac{1}{\sqrt{1-2 \alpha \sigma^{2}}} \int_{-\infty}^{\infty} \frac{\sqrt{1-2 \alpha \sigma^{2}}}{\sqrt{2 \pi} \sigma} e^{-\frac{x^{2}}{2 \sigma^{2}}\left(1-2 \alpha \sigma^{2}\right)} d x \\
& =\frac{1}{\sqrt{1-2 \alpha \sigma^{2}}} .
\end{aligned}
$$

Here we have used the observation that the integrand is the normal density with standard deviation $\sigma / \sqrt{1-2 \alpha \sigma^{2}}$.

We begin with the case when each entry of the projection matrix is chosen independently from the standard Normal distribution.

Lemma 2. Let $R=\left(r_{i j}\right)$ be a random $n \times k$ matrix, such that each entry $r_{i j}$ is chosen independently according to $N(0,1)$. For any vector fixed $u \in \boldsymbol{R}^{n}$, and any $\epsilon>0$, let $u^{\prime}=$ $\frac{1}{\sqrt{k}}\left(R^{T} u\right)$. Then, $\mathrm{E}\left(\left\|u^{\prime}\right\|^{2}\right)=\|u\|^{2}$ and

$$
\begin{gathered}
\operatorname{Pr}\left[\left\|u^{\prime}\right\|^{2}>(1+\epsilon)\|u\|^{2}\right] \leq\left((1+\epsilon) e^{-\epsilon}\right)^{k} \leq e^{-\left(\epsilon^{2}-\epsilon^{3}\right) \frac{k}{4}} \\
\operatorname{Pr}\left[\left\|u^{\prime}\right\|^{2}<(1-\epsilon)\|u\|^{2}\right] \leq\left((1-\epsilon) e^{\epsilon}\right)^{k} \leq e^{-\left(\epsilon^{2}-\epsilon^{3}\right) \frac{k}{4}}
\end{gathered}
$$


Proof: The expectation follows from a simple calculation. To obtain the bound on the concentration near the mean, let $X_{j}=\left(R_{j}^{T} \cdot u\right) /\|u\|$ and observe that

$$
X=\sum_{j=1}^{k} X_{j}^{2}=\sum_{j=1}^{k} \frac{\left(R_{j}^{T} \cdot u\right)^{2}}{\|u\|^{2}}
$$

where $R_{j}$ denotes the $j$ th column of $R$. Each $X_{j}$ has the standard normal distribution (since each component of $R_{j}$ does). Also note that

$$
\left\|u^{\prime}\right\|^{2}=\frac{\|u\|^{2}}{k} X
$$

Using Markov's inequality, we can then estimate the desired probability as

$$
\begin{aligned}
\mathrm{P}\left(\left\|u^{\prime}\right\|^{2} \geq(1+\epsilon)\|u\|^{2}\right)=\operatorname{Pr}(X \geq(1+\epsilon) k) & =\operatorname{Pr}\left(e^{\alpha X} \geq e^{(1+\epsilon) k \alpha}\right) \\
& \leq \frac{\mathrm{E}\left(e^{\alpha X}\right)}{e^{(1+\epsilon) k \alpha}} \\
& =\frac{\Pi_{j=1}^{k} \mathrm{E}\left(e^{\alpha X_{j}^{2}}\right)}{e^{(1+\epsilon) k \alpha}}=\left(\frac{\mathrm{E}\left(e^{\alpha X_{1}^{2}}\right)}{e^{(1+\epsilon) \alpha}}\right)^{k} .
\end{aligned}
$$

In the last line above, we have used the independence of the $X_{j}$ 's.

Similarly,

$$
\mathrm{P}\left(\left\|u^{\prime}\right\|^{2} \leq(1-\epsilon)\|u\|^{2}\right) \leq\left(\frac{\mathrm{E}\left(e^{-\alpha X_{1}^{2}}\right)}{e^{-(1-\epsilon) \alpha}}\right)^{k}
$$

`From Lemma 1,

$$
\mathrm{E}\left(e^{\alpha X_{1}^{2}}\right)=\frac{1}{\sqrt{1-2 \alpha}}
$$

for any $\alpha<\frac{1}{2}$. Thus we get,

$$
\operatorname{Pr}(X \geq(1+\epsilon) k) \leq\left(\frac{e^{-2(1+\epsilon) \alpha}}{(1-2 \alpha)}\right)^{\frac{k}{2}}
$$

The optimal choice of $\alpha$ is $\epsilon / 2(1+\epsilon)$. With this,

$$
\operatorname{Pr}(X \geq(1+\epsilon) k) \leq\left((1+\epsilon) e^{-\epsilon}\right)^{\frac{k}{2}} \leq e^{-\left(\epsilon^{2}-\epsilon^{3}\right) \frac{k}{4}} .
$$

Similarly,

$$
\operatorname{Pr}(X \leq(1-\epsilon) k) \leq\left(\frac{e^{2(1-\epsilon) \alpha}}{(1+2 \alpha)}\right)^{\frac{k}{2}} \leq\left((1-\epsilon) e^{\epsilon}\right)^{\frac{k}{2}} \leq e^{-\left(\epsilon^{2}-\epsilon^{3}\right) \frac{k}{4}}
$$

The main theorem of this section shows that this phenomenon is not specific to the Normal distribution. In the statement below, the condition that $\mathrm{E}\left(r^{2}\right)=1$ is for convenience. Instead 
one could have an arbitrary finite value $\sigma^{2}$ for this expectation, and scale the projection by $\sigma$.

Theorem 1. Let $R$ be a random $n \times k$ matrix, with each entry $r$ chosen independently from a distribution $\mathcal{D}$ that is symmetric about the origin with $\mathrm{E}\left(r^{2}\right)=1$. For any fixed vector $u \in \boldsymbol{R}^{n}$, let $u^{\prime}=\frac{1}{\sqrt{k}} R^{T} u$.

1. Suppose $B=\mathrm{E}\left(r^{4}\right)<\infty$. Then for any $\epsilon>0$,

$$
\mathrm{P}\left(\left[\left\|u^{\prime}\right\|^{2} \leq(1-\epsilon)\|u\|^{2}\right)\right] \leq e^{-\frac{\left(\epsilon^{2}-\epsilon^{3}\right) k}{2(B+1)}} .
$$

2. Suppose $\exists L>0$ such that for any integer $m>0, \mathrm{E}\left(r^{2 m}\right) \leq \frac{(2 m) !}{2^{m} m !} L^{2 m}$. Then for any $\epsilon>0$,

$$
\mathrm{P}\left(\left\|u^{\prime}\right\|^{2} \geq(1+\epsilon) L^{2}\|u\|^{2}\right) \leq\left((1+\epsilon) e^{-\epsilon}\right)^{k / 2} \leq e^{-\left(\epsilon^{2}-\epsilon^{3}\right) \frac{k}{4}} .
$$

Proof: Without loss of generality, assume that $\|u\|^{2}=1$. Let

$$
X_{i}=R_{i}^{T} u \quad \text { for } i=1, \ldots, k
$$

We have

$$
\mathrm{E}\left(X_{i}^{2}\right)=\mathrm{E}\left(\left(R_{i}^{T} u\right)^{2}\right)=\mathrm{E}\left(\left(\sum_{j=1}^{n} R_{i j} u_{j}\right)^{2}\right)=\sum_{j=1}^{n} \mathrm{E}\left(R_{i j}^{2}\right) u_{j}^{2}=1
$$

Then, if we define $Y$ as follows

$$
Y:=\sum_{i=1}^{k} X_{i}^{2}=k\left\|u^{\prime}\right\|^{2}, \quad \mathrm{E}(Y)=\sum_{i=1}^{k} \mathrm{E}\left(X_{i}^{2}\right)=k
$$

The deviation below the mean is relatively easy to bound, using the independence of the $X_{i}$ 's and Markov's inequality.

$$
\begin{aligned}
\mathrm{P}\left(\left\|u^{\prime}\right\|^{2}<(1-\epsilon)\|u\|^{2}\right) & =\mathrm{P}(Y<(1-\epsilon) k) \\
& =\mathrm{P}\left(e^{-\alpha Y}>e^{-\alpha(1-\epsilon) k}\right) \\
& \leq \frac{\mathrm{E}\left(e^{-\alpha Y}\right)}{e^{-\alpha(1-\epsilon) k}} \\
& =\left(\mathrm{E}\left(e^{-\alpha X_{1}^{2}}\right) e^{\alpha(1-\epsilon)}\right)^{k}
\end{aligned}
$$

and, using that $e^{-\alpha X_{1}^{2}} \leq 1-\alpha X_{1}^{2}+\alpha^{2} X_{1}^{4} / 2$, we get

$$
\mathrm{P}\left(\left\|u^{\prime}\right\|^{2}<(1-\epsilon)\|u\|^{2}\right) \leq\left(\left(1-\alpha \mathrm{E}\left(X_{1}^{2}\right)+\frac{\alpha^{2}}{2} \mathrm{E}\left(X_{1}^{4}\right)\right) e^{\alpha(1-\epsilon)}\right)^{k} .
$$


We can evaluate the moments easily: $\mathrm{E}\left(X_{1}^{2}\right)=1$ and, if we observe that the expectation of odd powers of $r$ is zero because of symmetry, we have (using the fact that $B \geq 1$ ),

$$
\begin{aligned}
\mathrm{E}\left(X_{1}^{4}\right) & =\mathrm{E}\left(\left(\sum_{j=1}^{n} R_{1 j} u_{j}\right)^{4}\right) \\
& =\sum_{j_{1}, j_{2}, j_{3}, j_{4}=1}^{n} \mathrm{E}\left(R_{1 j_{1}} R_{1 j_{2}} R_{1 j_{3}} R_{1 j_{4}}\right) u_{j_{1}} u_{j_{2}} u_{j_{3}} u_{j_{4}} \\
& =\sum_{j=1}^{n} \mathrm{E}\left(R_{1 j}^{4}\right) u_{j}^{4}+3 \sum_{j_{1} \neq j_{2}, j_{1}, j_{2}=1}^{n} \mathrm{E}\left(R_{1 j_{1}}^{2} R_{1 j_{2}}^{2}\right) u_{j_{1}}^{2} u_{j_{2}}^{2} \\
& \leq B \sum_{j=1}^{n} u_{j}^{4}+3 \sum_{j_{1} \neq j_{2}, j_{1}, j_{2}=1}^{n} u_{j_{1}}^{2} u_{j_{2}}^{2} \\
& \leq(B+2)\left(\sum_{j} u_{j}^{2}\right)^{2} \\
& =B+2 .
\end{aligned}
$$

Therefore, using the Taylor expansion of $e^{x}$, (in particular, $e^{-x+x^{2 / 2}} \geq 1-x$ for $x \geq 0$ and small enough).

$$
\begin{aligned}
\mathrm{P}\left(\left\|u^{\prime}\right\|^{2}<(1-\epsilon)\|u\|^{2}\right) & \leq\left(\left(1-\alpha+\frac{\alpha^{2}}{2}(B+2)\right) e^{\alpha(1-\epsilon)}\right)^{k} \\
& \leq\left(e^{-\alpha+\frac{\alpha^{2}(B+2)}{2}-\frac{1}{2}\left(\alpha-\frac{\alpha^{2}(B+2)}{2}\right)^{2}} e^{\alpha(1-\epsilon)}\right)^{k} \\
& \leq e^{-\frac{\left(\epsilon^{2}-\epsilon^{3}\right) k}{2(B+1)}}
\end{aligned}
$$

The last line above is obtained by setting $\alpha=\epsilon /(B+1)$ and noting that $B \geq 1$.

Similarly, for the deviation above the mean,

$$
\mathrm{P}\left(\left\|u^{\prime}\right\|^{2}>(1+\epsilon) L^{2}\|u\|^{2}\right) \leq\left(\frac{\mathrm{E}\left(e^{\alpha X_{1}^{2}}\right)}{e^{\alpha L^{2}(1+\epsilon)}}\right)^{k} .
$$

The main task is bounding $\mathrm{E}\left(e^{\alpha X_{1}^{2}}\right)$ from above using the assumptions of the theorem. This expectation is hard to evaluate directly since we don't know the distribution explicitly. However we have bounds on all the moments of $X_{1}^{2}$. Therefore, if we define a random variable $Z$ whose moments are all at least the moments of $X_{1}^{2}$, then $\mathrm{E}\left(e^{\alpha Z}\right)$ will be an upper bound on the required expectation. The following claim will be useful.

Claim 1. Let $f, g$ be distributions on $\boldsymbol{R}$ that are symmetric about the origin with the property that for any nonnegative integer $m, \mathrm{E}\left(Y^{2 m}\right) \leq \mathrm{E}\left(Z^{2 m}\right)$ where $Y, Z$ are drawn from $f, g$ respectively. Let $Y_{1}, \ldots, Y_{n}$ be i.i.d. from $f, Z_{1}, \ldots, Z_{n}$ be i.i.d from $g$. Then for any $u \in \boldsymbol{R}^{n}$, the random variables $\hat{Y}=\sum_{j=1}^{n} u_{j} Y_{j}$ and $\hat{Z}=\sum_{j=1}^{n} u_{j} Z_{j}$ satisfy $\mathrm{E}\left((\hat{Y})^{2 m}\right) \leq \mathrm{E}\left((\hat{Z})^{2 m}\right)$ for every nonnegative integer $m$. 
The claim is easy to prove. Compare the expectations of individual terms of $(\hat{Y})^{2 m}$ and $(\hat{Z})^{2 m}$. Since $Y_{i}, Z_{i}$ are symmetric about the origin, all terms in which they appear with an odd power have an expectation of zero. For any term in which all powers are even, by the assumption, the term from $\mathrm{E}\left((\hat{Z})^{2 m}\right)$ dominates.

To apply this to our setting, we know that

$$
X_{1}=\sum_{j=1}^{n} u_{j} r_{j}
$$

where each $r_{j}$ is drawn from the given distribution $\mathcal{D}$. Define

$$
Y_{1}=\sum_{j=1}^{n} u_{j} r_{j}^{\prime}
$$

where each $r_{j}^{\prime}$ is drawn from $N(0, L)$. Then for all $j$, and any integer $m>0$,

$$
\mathrm{E}\left(r_{j}^{2 m}\right) \leq \frac{(2 m) !}{2^{m} m !} L^{2 m}=\mathrm{E}\left(\left(r_{j}^{\prime}\right)^{2 m}\right)
$$

using the well-known formula for the moments of $N(0, L)$. So, $\mathrm{E}\left(X_{1}^{2 m}\right) \leq \mathrm{E}\left(Y_{1}^{2 m}\right)$. Moreover, the distribution of $Y_{1}$ is $N(0, L)$. Therefore,

$$
\mathrm{E}\left(e^{\alpha X_{1}^{2}}\right) \leq \mathrm{E}\left(e^{\alpha Y_{1}^{2}}\right)=\frac{1}{\sqrt{1-2 \alpha L^{2}}} .
$$

Using this,

$$
\mathrm{P}\left(\left\|u^{\prime}\right\|^{2}>(1+\epsilon) L^{2}\|u\|^{2}\right) \leq\left(\frac{e^{-2 \alpha L^{2}(1+\epsilon)}}{1-2 \alpha L^{2}}\right)^{\frac{k}{2}}
$$

The optimal choice of $\alpha$ is $\epsilon / 2 L^{2}(1+\epsilon)$, and we get that for any $\epsilon>0$,

$$
\mathrm{P}\left(\left\|u^{\prime}\right\|^{2}>(1+\epsilon) L^{2}\|u\|^{2}\right) \leq\left((1+\epsilon) e^{-\epsilon}\right)^{\frac{k}{2}} \leq e^{-\left(\epsilon^{2}-\epsilon^{3}\right) \frac{k}{4}} .
$$

The last inequality was obtained by using the inequality $\ln (1+\epsilon) \leq \epsilon-\epsilon^{2} / 2+\epsilon^{3} / 2$.

Corollary 1. If every entry of an $n \times k$ matrix $R$ is chosen according to $U(-1,1)$, then for any fixed vector $u \in \boldsymbol{R}^{n}$ and any $\epsilon>0$, the vector $u^{\prime}=\frac{1}{\sqrt{k}} R^{T} u$ satisfies

$$
\mathrm{P}\left(\left\|u^{\prime}\right\|^{2} \geq(1+\epsilon)\|u\|^{2}\right) \leq e^{-\left(\epsilon^{2}-\epsilon^{3}\right) \frac{k}{4}} \text { and } \mathrm{P}\left(\left\|u^{\prime}\right\|^{2} \leq(1-\epsilon)\|u\|^{2}\right) \leq e^{-\left(\epsilon^{2}-\epsilon^{3}\right) \frac{k}{4}} .
$$

Proof: For $r$ drawn from $U(-1,1), \mathrm{E}\left(r^{2 m}\right)=1$ for any integer $m>0$. Therefore, we can apply Theorem 1 with $L=B=1$ to get the conclusion of the corollary. 
Let $R$ be an $n \times k$ matrix whose entries are chosen independently from either $N(0,1)$ or $U(-1,1)$, independently. The following theorem summarizes the results of this section. Alternative proofs for the case of $N(0,1)$ appeared in Indyk and Motwani (1998) and DG.

Theorem 2 (Neuronal RP). Let $u, v \in \boldsymbol{R}^{n}$. Let $u^{\prime}$ and $v^{\prime}$ be the projections of $u$ and $v$ to $\boldsymbol{R}^{k}$ via a random matrix $R$ whose entries are chosen independently from either $N(0,1)$ or $U(-1,1)$. Then,

$$
\mathrm{P}\left[(1-\epsilon)\|u-v\|^{2} \leq\left\|u^{\prime}-v^{\prime}\right\|^{2} \leq(1+\epsilon)\|u-v\|^{2}\right] \geq 1-2 e^{-\left(\epsilon^{2}-\epsilon^{3}\right) \frac{k}{4}} .
$$

Proof: Apply Theorem 1 to the vector $u-v$.

We conclude this section with a useful corollary. A similar proof can be found in Ben-David, et al. (2002).

Corollary 2. Let $u, v$ be vectors in $\boldsymbol{R}^{n}$ s.t. $\|u\|,\|v\| \leq 1$. Let $R$ be a random matrix whose entries are chosen independently from either $N(0,1)$ or $U(-1,1)$. Define $u^{\prime}=\frac{1}{\sqrt{k}} R^{T} u$ and $v^{\prime}=\frac{1}{\sqrt{k}} R^{T} v$. Then for any $\epsilon>0$,

$$
\mathrm{P}\left(u \cdot v-c \leq u^{\prime} \cdot v^{\prime} \leq u \cdot v+c\right) \geq 1-4 e^{-\left(\epsilon^{2}-\epsilon^{3}\right) \frac{k}{4}} .
$$

Proof: Applying Theorem 2 to the vectors $u, v$ and $u-v$, we have that with probability at least $1-4 e^{-\left(c^{2}-c^{3}\right) \frac{k}{4}}$,

$$
\text { and } \quad \begin{aligned}
& (1-c)\|u-v\|^{2} \leq\left\|u^{\prime}-v^{\prime}\right\|^{2} \leq(1+c)\|u-v\|^{2} \\
& (1-c)\|u+v\|^{2} \leq\left\|u^{\prime}+v^{\prime}\right\|^{2} \leq(1+c)\|u+v\|^{2} .
\end{aligned}
$$

Then,

$$
\begin{aligned}
4 u^{\prime} \cdot v^{\prime} & =\left\|u^{\prime}+v^{\prime}\right\|^{2}-\left\|u^{\prime}-v^{\prime}\right\|^{2} \\
& \geq(1-c)\|u+v\|^{2}-(1+c)\|u-v\|^{2} \\
& =4 u \cdot v-2 c\left(\|u\|^{2}+\|v\|^{2}\right) \\
& \geq 4 u \cdot v-4 c .
\end{aligned}
$$

Thus $u^{\prime} \cdot v^{\prime} \geq u \cdot v-c$. The other inequality is similar.

In what follows, we will apply random projection by picking entries of the projection matrix independently from $N(0,1)$ or $U(-1,1)$. We remark that one could use other distributions via Theorem 1. 


\section{Learning efficiently by reducing dimensionality}

In this section, we describe learning algorithms for robust concepts and derive bounds on the number of examples required and the running times. Our bounds will be functions of the robustness parameter $l$, and the $\epsilon, \delta$ learning parameters, but will be independent of the actual number of attributes of the concept class.

We are given labelled examples from an unknown distribution $\mathcal{D}$. The generic algorithm for learning robust concepts is based on the following two high-level ideas:

1. Since the target concept is robust, random projection of the examples to a much lower-dimensional subspace will "preserve" the concept.

2. In the lower-dimensional space, the number of examples and the time required to learn concepts are relatively small.

Before applying this approach to specific concept classes, we recall some fundamental theorems in learning theory. For the concept class $\mathcal{C}$ under consideration, let $C(m, k)$ denote the maximum number of distinct labellings of $m$ points that can be obtained by using concepts from $\mathcal{C}$ in $\boldsymbol{R}^{k}$. The following well-known theorem (see Kearns \& Vazirani (1994) or Blumer et al. (1989)) gives a bound on the size of the sample so that a hypothesis that is consistent with the sample also has, with high probability, small error with respect to the entire distribution.

Theorem 3. Let $\mathcal{C}$ be any concept class in $\boldsymbol{R}^{k}$. Let $w$ be a concept from $\mathcal{C}$ that is consistent with $m$ labelled examples of some concept in $C$. Then with probability at least $1-\delta, w$ correctly classifies at least $(1-\epsilon)$ fraction of $\mathcal{D}$ provided

$$
m>\frac{4}{\epsilon} \log C(2 m, k)+\frac{4}{\epsilon} \log \frac{2}{\delta} .
$$

The notion of VC-dimension (Vapnik \& Chervonenkis, 1971) is closely connected to the number of distinct labelings as expressed in the following basic theorem.

Theorem 4 (Blumer et al. 1989). Let $C$ be a concept class of VC-dimension d. Then, the number of distinct labelings of $m$ points by concepts in $C$ is at most

$$
C[m] \leq \sum_{i=0}^{d}\left(\begin{array}{c}
m \\
i
\end{array}\right) .
$$

If the algorithm finds a hypothesis that is nearly consistent with the sample (rather than fully consistent as in the previous theorem), this too generalizes well. The number of samples required increases by a constant factor. The theorem below is a slight variant of a similar theorem from Blumer et al. (1989). We give a self-contained proof in the appendix for the reader's convenience.

Theorem 5. For $\epsilon \leq 1 / 4$, let $w$ be a concept from $\mathcal{C}$ in $\boldsymbol{R}^{k}$ that correctly classifies at least $a(1-\epsilon / 8)$ fraction of a sample of $m$ points drawn from $\mathcal{D}$ such that

$$
m \geq \frac{32}{\epsilon} \log C(2 m, k)+\frac{32}{\epsilon} \log \frac{2}{\delta} .
$$


Then with probability at least $1-\delta, w$ correctly classifies at least a $1-\epsilon$ fraction of $\mathcal{D}$.

\subsection{Half-spaces}

We begin with the problem of learning a half-space in $\boldsymbol{R}^{n}$ (a linear threshold function). This is one of the oldest problems studied in learning theory. The problem can be solved in polynomial-time by using an algorithm for linear programming on a sample of $O(n)$ examples (note that this is not a strongly polynomial algorithm-its complexity depends only polynomially on the number of bits in the input). Typically, however, it is solved by using simple greedy methods. A commonly-used greedy algorithm is the Perceptron Algorithm (Agmon, 1954; Rosenblatt, 1962), which has the following guarantee: Given a collection of data points in $\boldsymbol{R}^{n}$, each labeled as positive or negative, the algorithm will find a vector $w$ such that $w \cdot x>0$ for all positive points $x$ and $w \cdot x<0$ for all negative points $x$, if such a vector exists. ${ }^{1}$ The running time of the algorithm depends on a separation parameter (described below). However, in order for the hypothesis to be reliable, we need to use a sample of $\Omega(n)$ points, since the VC-dimension of half-spaces in $\boldsymbol{R}^{n}$ is $n+1$.

Let $\mathcal{H}_{n}$ be the class of homogenous half-spaces in $\boldsymbol{R}^{n}$. Let $(h, \mathcal{D})$ be a concept-distribution pair such that the half-space $h \in \mathcal{H}_{n}$ is $\ell$-robust with respect to the distribution $\mathcal{D}$ over $\boldsymbol{R}^{n}$. We restrict $\mathcal{D}$ to be over the unit sphere (i.e., all the examples are at unit distance from the origin). The latter condition is not really a restriction since examples can be scaled to have unit length without changing their labels. The parameters $k$ and $m$ in the algorithm below will be specified later.

\section{Half-space Algorithm:}

1. Choose an $n \times k$ random matrix $R$ by picking each entry independently from $N(0,1)$ or $U(-1,1)$.

2. Obtain $m$ examples from $\mathcal{D}$ and project them to $\boldsymbol{R}^{k}$ using $R$.

3. Run the following Perceptron Algorithm in $\boldsymbol{R}^{k}$ : Let $w=0$. Perform the following operation until all examples are correctly classified:

Pick an arbitrary misclassified example $x$ and let $w \leftarrow w+\operatorname{label}(x) x$.

4. $\quad$ Output $R$ and $w$.

A future example $x$ is labelled positive if $w \cdot\left(R^{T} x\right) \geq 0$ and negative otherwise. This is of course the same as checking if $\left(w R^{T}\right) \cdot x>0$, i.e., a half-space in the original $n$-dimensional space.

We can assume that $h$, the normal vector to the concept half-space, is of unit length. The idea behind the algorithm is that when $k$ is large enough, in the $k$-dimensional subspace obtained by projection, the half-space through the origin defined by $R^{T} h$, i.e., $\left(R^{T} h\right) \cdot y \geq 0$, classifies most of the projected distribution correctly. We will show that in fact this halfspace remains robust with respect to a projected sample of sufficiently large size. To find a consistent half-space, we use the classical perceptron algorithm. It is well-known (see Minsky \& Papert (1969)) that the convergence of this algorithm depends on the margin, i.e., in our terminology, the robustness of the target half-space.

Theorem 6. (Minsky \& Papert, 1969) Suppose the data set $S$ can be correctly classified by some unit vector $w$. Then, the Perceptron Algorithm converges in at most $1 / \sigma^{2}$ iterations,

\footnotetext{
${ }^{1}$ A zero threshold can be achieved by adding an extra dimension to the space.
} 
where

$$
\sigma=\min _{x \in S} \frac{|w \cdot x|}{\|x\|}
$$

For an $\ell$-robust half-space, we have $\sigma \geq \ell$. The theorem says that the perceptron algorithm will find a consistent half-space in at most $1 / \ell^{2}$ iterations. We can now state and prove the main result of this section.

Theorem 7. An $\ell$-robust half-space in $\boldsymbol{R}^{n}$ can be $(\epsilon, \delta)$-learned by projecting a set of $m$ examples to $\boldsymbol{R}^{k}$ where

$$
k=\frac{100}{\ell^{2}} \ln \frac{100}{\epsilon \ell \delta}, \quad m=\frac{8 k}{\epsilon} \log \frac{48}{\epsilon}+\frac{4}{\epsilon} \log \frac{4}{\delta}=O\left(\frac{1}{\ell^{2}} \cdot \frac{1}{\epsilon} \cdot \ln \frac{1}{\epsilon} \ln \frac{1}{\epsilon \ell \delta}\right)
$$

in time $n \cdot \operatorname{poly}\left(\frac{1}{\ell}, \frac{1}{\epsilon}, \log \frac{1}{\delta}\right)$ time.

Proof: For an example point $x$, we let $x^{\prime}$ denote its projection. We let $h^{\prime}$ denote the projection of $h$, the normal to the target half-space. We would like the following events to occur (by the choice of the projection matrix $R$ ):

1. For each example $x$, its projection $x^{\prime}$ has length at most $1+\frac{\ell}{2}$. Similarly, $\left\|h^{\prime}\right\| \leq 1+$ $\frac{\ell}{2}$.

2. For each example $x$, if $h \cdot x \geq \ell$, then $h^{\prime} \cdot x \geq \frac{\ell}{2}$; if $h \cdot x \leq-\ell$, then $h^{\prime} \cdot x^{\prime} \leq-\frac{\ell}{2}$.

We now bound the probability that one of these events does not occur. For any single example $x$, applying Corollary 2 with $\epsilon=\ell / 2$ and our choice of $k$, the probability that $\left\|x^{\prime}\right\|>1+\frac{\ell}{2}$ is at most

$$
e^{-\left(\frac{\ell^{2}}{4}-\frac{\ell^{3}}{8}\right) \frac{k}{4}} \leq e^{-\frac{\ell^{2} k}{32}} \leq\left(\frac{\epsilon \ell \delta}{100}\right)^{\frac{100}{32}}<\frac{\delta}{4(m+1)}
$$

Adding this up over all the $m$ examples and the vector $h$, we get a failure probability of at most $\delta / 4$.

Next, by Corollary 2, with $u=h$ and $v=x$, the probability that the second event does not occur for any particular example $x$ is at most $\delta / 4 m$. Again this contributes a total failure probability of at most $\delta / 4$. Thus, both events occur with probability at least $1-\delta / 2$.

These events imply that the half-space in $\boldsymbol{R}^{k}$ defined by $h^{\prime}$ correctly classifies all the $m$ examples after projection (with probability at least $1-\delta / 2$ ). Moreover, after scaling the examples to have length at most 1 , the margin is at least

$$
\sigma \geq \frac{\ell / 2}{1+\frac{\ell}{2}} \geq \frac{\ell}{3}
$$

Now, by Theorem 6 , the perceptron algorithm will find a consistent half-space in $9 / \ell^{2}$ iterations.

Finally, we need to show that $m$ is large enough that hypothesis found generalizes well. We will apply Theorem 3 to half-spaces through the origin in $\boldsymbol{R}^{k}$. The VC-dimension of the 笔Springer 
latter concept class is $k$ and so, by Theorem 4, we get the following well-known bound on the number of distinct half-spaces (see e.g. Kearns \& Vazirani (1994)):

$$
C(2 m, k) \leq \sum_{i=0}^{k-1}\left(\begin{array}{c}
2 m \\
i
\end{array}\right) \leq\left(\frac{2 e m}{k}\right)^{k}
$$

Our choice of $m$ satisfies

$$
m=\frac{8 k}{\epsilon} \log \frac{48}{\epsilon}+\frac{4}{\epsilon} \log \frac{4}{\delta}>\frac{4}{\epsilon} \log C(2 m, k)+\frac{4}{\epsilon} \log \frac{4}{\delta} .
$$

Therefore, applying Theorem 3 with $\delta / 2$ in place of $\delta$, the half-space found by the algorithm correctly classifies at least $1-\epsilon$ of the original distribution with probability at least $1-\delta / 2$. This gives an overall success probability of at least $1-\delta$.

The perceptron algorithm and its variants are known to be resistant to various types of random classification noise (Bylander, 1994; Blum et al., 1996). It is a straightforward consequence that these properties continue to hold for the algorithm described here. In the concluding section, we discuss straightforward bounds for agnostic learning.

\subsection{Intersections of half-spaces}

The next problem we consider is learning an intersection of $t$ half-spaces in $\boldsymbol{R}^{n}$, i.e., the positive examples all lie in the intersection of $t$ half-spaces and the negative examples lie outside that region. It is not known how to solve the problem for an arbitrary distribution. However efficient algorithms have been developed for reasonably general distributions assuming that the number of half-spaces is relatively small (Blum \& Kannan, 1993; Vempala, 2004). Here, we derive efficient learning algorithms for robust concepts in this class.

We assume that all the half-spaces are homogenous. Let the concept class of intersections of half-spaces be denoted by $\mathcal{H}(t, n)$. A single concept in this class is specified by a set of $t$ half-spaces $P=\left\{h_{1}, \ldots, h_{t}\right\}$, and the positive examples are precisely those that satisfy $h_{i} \cdot x \geq 0$ for $i=1 \ldots t$. Let $(P, \mathcal{D})$ be a concept-distribution pair such that $P$ is $\ell$-robust with respect to the distribution $\mathcal{D}$. We assume that the support $\mathcal{D}$ is a subset of the unit sphere (and remind the reader that this as well as homogeneity are not really restrictive, as they can be achieved by scaling and adding an extra dimension, respectively; see e.g. (Vempala, 2004)).

Let denote $C(m, t, k)$ denote the maximum number of distinct labellings of $m$ examples in $R^{k}$ using concepts from $\mathcal{H}(t, k)$. Then,

$$
C(2 m, t, k) \leq\left(\sum_{i=0}^{k-1}\left(\begin{array}{c}
2 m \\
i
\end{array}\right)\right)^{t} \leq\left(\frac{2 e m}{k}\right)^{t k}
$$

This can be seen as follows: For $t=1$, this is just (1), the number of ways to assign + or -1 to $2 m$ points using a half-space. If we give each point $t$ labels, one for each of $t$ halfspaces, then the total number of possible labellings is the middle term in (2). We consider two labellings distinct iff the subset of points that are labelled + by all $t$ half-spaces is different. Thus the total number of distinct labellings by $t$ half-spaces can only be smaller than this bound. 
Given $m$ examples, we can always find a consistent hypothesis (if one exists) using a brute-force algorithm that enumerates all the combinatorially distinct half-spaces and pick $t$ of them (with replacement). We apply this to learning a robust intersection of $t$-half-spaces after projecting a sufficiently large sample to a lower-dimensional subspace. The parameters $k$ and $m$ below will be specified shortly.

\section{$t$-Half-spaces Algorithm:}

1. Choose an $n \times k$ random matrix $R$ for projection by choosing each entry independently from $N(0,1)$ or $U(-1,1)$.

2. Obtain $m$ examples from $\mathcal{D}$ and project them to $\boldsymbol{R}^{k}$ using $R$.

3. Find a hypothesis $Q=\left\{w_{1}, \ldots, w_{t}\right\}$ where each $w_{i} \in \boldsymbol{R}^{k}$ such that the intersection of the half-spaces $w_{i} \cdot x \geq 0$ for $i=1, \ldots, t$ is consistent with the labels of the projected examples.

4. $\quad$ Output $R$ and $Q$.

A future example $x$ is projected down as $R^{T} x$ and labelled according to $Q$, i.e., it is positive if $w_{i} \cdot\left(R^{T} x\right) \geq 0$ for all $i=1, \ldots, t$.

Theorem 8. An $\ell$-robust intersection of thalf-spaces in $\boldsymbol{R}^{n}$ can be $(\epsilon, \delta)$-learned by projecting $m$ examples to $\boldsymbol{R}^{k}$ where

$$
k=\frac{100}{\ell^{2}} \ln \frac{100 t}{\epsilon \ell \delta} \quad \text { and } \quad m=\frac{8 k t}{\epsilon} \log \frac{48 t}{\epsilon}+\frac{4}{\epsilon} \log \frac{4}{\delta}=O\left(\frac{t}{\epsilon \ell^{2}} \log \frac{t}{\epsilon} \log \frac{t}{\ell \epsilon \delta}\right)
$$

in time $O(n m k)+\left(\frac{48 t}{\epsilon} \log \frac{4 t}{\epsilon \delta}\right)^{k t}$.

Proof: The proof is similar to that of Theorem 7 and we only sketch it.

Let the original set of half-spaces be $h_{1} \cdot x \geq 0, \ldots, h_{t} \cdot x \geq 0$, where each $h_{i}$ is a unit vector in $\boldsymbol{R}^{n}$. We consider the projections of these, $h_{i}^{\prime}=\frac{1}{\sqrt{k}} R^{T} h_{i}$, and the following events: For each example $x$ and normal vector $h_{i}$, if $h_{i} \cdot x \geq \ell$, then $h_{i}^{\prime} \cdot x^{\prime}>0$; If $h_{i} \cdot x \leq-\ell$, then $h_{i}^{\prime} \cdot x^{\prime}<0$.

For our choice of $k$ and $m$, it follows from Corollary 2 that these events all happen with probability at least $1-\delta / 2$. Therefore, after projection, with this probability, there is a hypothesis from $\mathcal{H}(t, k)$ that is consistent with all $m$ examples. Using Theorem 3 along with (2), it follows that any hypothesis consistent with a sample of size

$$
m=\frac{8 k t}{\epsilon} \log \frac{2 t}{\epsilon}+\frac{4}{\epsilon} \log \frac{4}{\delta}
$$

will correctly classify $(1-\epsilon)$ of the distribution with probability at least $1-\delta / 2$. This gives an overall success probability of at least $1-\delta$. The running time of the enumerative algorithm is $O\left((2 e m / k)^{k t}\right)$.

If $t, \ell, \epsilon, \delta$ are all constant, then the algorithm runs in linear time. If only $\ell, \epsilon, \delta$ are constant, then the algorithm has running time $O\left(n t \log ^{3} t\right)+(t \log t)^{O(t \log t)}$. This is significantly faster than the best-known algorithms for the general case (see Section 1.1 for recent improvements). Both results do not need any further assumptions on the distribution $\mathcal{D}$ besides 
robustness. Previous algorithms for the problem assumed that $\mathcal{D}$ was either symmetric (Baum, 1990), uniform (Blum \& Kannan, 1993) or non-concentrated (Vempala, 1997). Recently, an improved time complexity for learning robust intersections of half-spaces was obtained in Klivans and Servedio (2004) using an algorithm for learning polynomial threshold functions in the projected space in place of the enumerative algorithm used here. The improvement in the time complexity comes along with a substantial increase in the sample complexity.

\subsection{Balls}

Finally, we briefly discuss the concept class of balls in $\boldsymbol{R}^{n}$, illustrating how robustness plays a role in learning nonlinear concepts.

A Ball $B\left(x_{0}, r\right)$ in $\boldsymbol{R}^{n}$ is defined as

$$
B\left(x_{0}, r\right)=\left\{x \in \boldsymbol{R}^{n}:\left\|x-x_{0}\right\| \leq r\right\}
$$

where $x_{0}$ (the center) is a fixed point in $\Re^{n}$ and $r$ (the radius) is a fixed real value. The set of points in $B\left(x_{0}, r\right)$ are labelled positive and those outside are labelled negative.

It is well-known that the VC-dimension of balls in $\boldsymbol{R}^{n}$ is $n+1$ and so the number of examples required to $(\epsilon, \delta)$-learn a ball is $O\left(\frac{n}{\epsilon} \log \frac{1}{\epsilon}+\frac{1}{\epsilon} \log \frac{1}{\delta}\right)$. How many examples do we need to learn an $\ell$-robust ball? The following theorem follows easily from the neuronal projection theorem.

Theorem 9. An $\ell$-robust ball of radius in $\boldsymbol{R}^{n}$ of radius at most 1 can be $(\epsilon, \delta)$-learned by projecting $m$ examples to $\boldsymbol{R}^{k}$ where

$$
k=\frac{100}{\ell^{2}} \ln \frac{100}{\epsilon \ell \delta} \quad \text { and } \quad m=\frac{8 k}{\epsilon} \log \frac{48}{\epsilon}+\frac{4}{\epsilon} \log \frac{4}{\delta}
$$

and then finding a ball in $\boldsymbol{R}^{k}$ consistent with the projected examples.

Proof: With probability 1, any positive example $x$ drawn from the distribution $\mathcal{D}$ will satisfy

$$
\left\|x-x_{0}\right\| \leq r-l
$$

while any negative example $x$ will satisfy

$$
\left\|x-x_{0}\right\| \geq r+l
$$

Using Theorem 2 with our choice of $k$ and $\epsilon=\ell / 2$, for any one $x$, its projection $x^{\prime}$ satisfies

$$
\left(1-\frac{\ell}{2}\right)\left\|x-x_{0}\right\| \leq\left\|x^{\prime}-x_{0}^{\prime}\right\| \leq\left(1+\frac{\ell}{2}\right)\left\|x-x_{0}\right\|
$$

with probability at least $1-\frac{\delta}{2 m}$. So, with probability $1-\delta / 2$, all the projected examples satisfy the above inequality. Further, since the radius of the concept ball is at most 1 ,

$$
\left\|x-x_{0}\right\|+\frac{\ell}{2} \leq\left\|x^{\prime}-x_{0}^{\prime}\right\| \leq\left\|x-x_{0}\right\|+\frac{\ell}{2} .
$$


Thus, the ball $B\left(x_{0}^{\prime}, r\right)$ in $\boldsymbol{R}^{k}$ is consistent with the projected examples and the theorem follows. Finally, we can use Theorem 3 to verify that $m$ is large enough for this to be an $(\epsilon, \delta)$-learning algorithm.

\subsection{Noise tolerance}

Here we note that the algorithms can be adapted to be resistant to malicious classification noise (agnostic learning). In a sample of $s$ examples, let the labels of at most $\gamma s$ of them be corrupted arbitrarily. Fix a hypothesis class $H$ and let $f(\ell)$ be the bound on the number of examples required to learn concepts with robustness $\ell$. Then to deal with this noise "rate" $\gamma$, we obtain $f(\ell) /(1-\gamma)$ examples, and for every subset of size $f(\ell)$ of the sample, we run the learning algorithm for the hypothesis class and output a hypothesis that correctly classifies the subset. The total number of runs of the algorithms is at most $2^{2 f(\ell)}$. So, for example, half-spaces in $\boldsymbol{R}^{n}$ can be learned in $\operatorname{poly}(n)$ time for robustness as low as $\sqrt{\frac{\log \log n}{\log n}}$. Another way to interpret this is that we can find hypothesis that minimize the number of mistakes. This was observed by Avrim Blum.

\section{Discussion}

\subsection{A robust model of categorization}

The model studied in this paper can be viewed as a rudimentary model of human learning. In this model, at the outer level of processing, there is a layer of neurons that produces a random summary of any stimuli presented. It is the summary that is then processed by learning algorithms. The outer level plays the role of random projection. The main insight of the model is that even a random summary that is independent of any specific concept and independent of the distribution on examples (stimuli), can preserve the essential elements necessary for learning the category. The ease of learning and the extent to which the summary preserves the concepts depends on their robustness-the more robust a concept, the shorter a summary needs to be and the easier it is to learn. In this section, we draw from work in cognitive and neuropsychology to see how the predictions of this model hold up. Our model goes beyond previous ones that made similar predictions in suggesting a simple physiological mechanism.

An interesting prediction of our model is that learning concepts that are more robust requires fewer examples. This prediction is supported by many psychological studies (Glass, et al., 1979; Komatsu, 1992; Reed, 1982; Reed \& Friedman, 1973; Rosch, 1978; Rosch et al., 1976), in particular those that refer to concept formation as stemming from the family resemblence perspective (for a detailed account of other prominent views, see (Komatsu, 1992; Rakinson \& Oakes, 2003)). The family resemblence perspective argues that categories (concept classes) as formed by humans are hierarchical (Reed, 1982), with three clear levels called the Superordinate, Basic and Subordinate. For example, for the Superordinate category of Mammals, some Basic level categories are Elephant, Dog, Human, and the Subordinate categories for Elephant would be African Elephant and Indian Elephant. Similarly, the Superordinate category of Musical Instruments has below it the Basic level categories of Guitar, Piano, Drum, etc. and Guitar, has below it Subordinate categories such as Folk Guitar and Steel Guitar. The Basic level categories are considered the most important, and are the most clearly demarcated from each other. In our terminology they are the most robust, and we 
expect them to be easier to learn. This is indeed the case as noted by Rosch et al. (1976), "...basic level categories are the most differentiated from one another and they are therefore the first categories we learn and the most important in language."

A related theory of how humans form categories is based on the notion of Prototypes (Glass, et al., 1979; Komatsu, 1992). The essential predictions of this theory can also be derived from robustness. Prototypes represent the most typical members of a category. The theory says that we abstract a prototype for a category by forming some weighted average of (a subset of) the defining features of examples from the category. A subsequent instance is compared to the prototype, and if it has a sufficient degree of similarity to the prototype then it is judged to be a member of the category. This explains the results of studies where it is found that when asked to list examples of a category, subjects consistently list members that are closer to the prototype both earlier and more often (e.g., for the category Bird, the examples Sparrow and Robin are produced more often than Ostrich) Rosch (1978). Further when asked to classify instances, it is found that examples closer to the prototype are classified more quickly. Similar results were found in studies with artificially generated categories (Reed \& Friedman, 1973).

For a prototype $P$, we could define a family of nested concepts within the category of $P$ according to the distance from $P$. Then the members of the innermost concept are very similar to $P$, the members of the next concept are a bit more varied, and the variation increases as the maximum distance of the concepts from $P$ grows. In other words the innermost concept is the most robust in terms of demarcating the category from objects that are not members (of the family category), and the robustness decreases as we move outward. The arguments in this paper imply that the inner concepts would be easier to learn and label than the outer ones. This is exactly what was observed in the aforementioned studies.

In our model as the organism is presented with a given stimulus, a random summary of its characteristics is captured. After another member of the same family is observed another summary of characteristics is abstracted. The characteristics that are shared among stimuli from the same "family" would in time lead to a set of summaries with analogous characteristics. These analogous characteristics would give rise to a protypical family member (say Robin for the bird family) because it embodies many of the characteristics which have a greater probability of appearing in these "random summaries" since they are more common in the family (small, feathers, flies) and not other characteristics which are likely to be atypical (large, does not fly for Ostrich or has no feathers for Penguin).

Another question that our model addresses concerns the need to make distinctions between perceptual (red, square, loud) or conceptual categories (dessert vs. salad or good vs. evil). This issue is prominent in current research on categorization (Mandler, 2003). While the examples we have mentioned (birds, elephants and guitars) can be described as perceptual (objectbased) categories, it is worth noting that our model also applies to conceptual categories. The idea is that along with physical characteristics, abstract characteristics (that are also ultimately functions of the stimuli, e.g. "soulfulness" might include Steel Guitar and Saxophone) are preserved by the random summaries. The predictions of our model are similar to those of the family resemblance view; the latter has been successfully used to go beyond object-based categores to psychological phenomena such as emotions and personality traits (Komatsu, 1992). At the outer-level mechanism of our model, there is no need for separate learning systems for categorization.

In the same vein, the current model also speaks to the broad neuropsychological issue of whether it is necessary to propose a multiple-system (i.e., various brain regions) model as opposed to a single-system model (general brain processing) to account for object recognition on the one hand and categorization on the other (Knowlton, 1999). We see our model as a 
single-system model that provides a general physiological "outer-level” for learning. As such we believe this model partially answers the call to "develop formalized single-system and multiple-system mathematical models of categorization, to collect rich sets of parametric data using both normal and brain-damaged population and to test the ability of the respective models to account quatitatively for the data.” (Nosofsky \& Zaki, 1969).

\subsection{Open problems}

In the discrete setting, an $\ell$-robust concept is one where a positive example retains its label even if an $\ell$-fraction of its attributes are changed. An important open problem in computational learning theory is that of learning DNF formulae from the uniform distribution without membership queries. This concept class can be viewed as intersections of half-spaces with robustness $1 / \sqrt{n}$. If there is an algorithm for learning DNF formulae in time polynomial in $n$ and $1 / \ell$, this would solve the problem of learning DNF formulae without membership queries.

We have seen how robustness reduces the learning complexity of some important concept classes. We conclude with the following questions: What are concept classes for which robustness does not reduce learning complexity? In particular, what is the complexity of learning robust polynomial threshold functions?

\section{Appendix}

Proof: (of Theorem 5.) We basically mimic the proof of the fundamental VC theorem. The only difference is that in that theorem, it is assumed that there is a hypothesis consistent with the entire sample. Here we can only assume that there is a hypothesis that correctly classifies $1-\epsilon / 4$ fraction of the sample.

Let us call a hypothesis a bad hypothesis if it has error more than $\epsilon$ on the distribution. Let $A$ be the event that there exists a bad consistent hypothesis, i.e., a hypothesis that has error less than $\epsilon / 8$ on the sample and error greater than $\epsilon$ on the distribution. We would like to show that the probability of event $A$ is at most $\delta$. To do this, we define $B$ to be the event that for a sequence of $2 m$ examples, there is a concept that has error less than $\epsilon / 8$ on the first $m$ and greater than $\epsilon / 2$ on the remaining $m$.

Next we observe that $\operatorname{Pr}(A) \leq 2 \cdot \operatorname{Pr}(B)$. This is because

$$
\operatorname{Pr}(B) \geq \operatorname{Pr}(A \text { and } B)=\operatorname{Pr}(A) \cdot \operatorname{Pr}(B / A)
$$

The probability of $B$ given $A, \operatorname{Pr}(B / A)$ is the probability that a hypothesis that has error $\epsilon$ on the distribution has error at least $\epsilon / 2$ on a set of $m$ examples. Using Chebychev's inequality, this latter probability is at least $1 / 2$.

To complete the proof we will bound the probability of $B$. Fix any set of $2 m$ examples and consider a random partition of them into two equal-sized sets $S_{1}$ and $S_{2}$. Let $\hat{h}$ be a hypothesis which disagrees with the target hypothesis on at least $\epsilon m / 2$ of the $2 m$ examples. This is a candidate for causing event $B$.

Let $X_{i}$, for $i=1, \ldots, m$ denote the event that $\hat{h}$ makes an error on the $i$ 'th example in $S_{1}$. Then $E\left(X_{i}\right)=\epsilon / 4$. Define

$$
X=\sum_{i=1}^{m} X_{i}
$$


Then $E(X)=\epsilon m / 4$. By Chernoff's inequality,

$$
\operatorname{Pr}\left(X \leq \frac{\epsilon}{4}(1-c)\right) \leq e^{-\frac{\epsilon m c^{2}}{8}}
$$

That is,

$$
\operatorname{Pr}\left(X \leq \frac{\epsilon}{8}\right) \leq e^{-\epsilon m / 32}
$$

The total number of distinct hypothesis for the set of $2 m$ examples is at most $C(2 m, k)$. In other words, this is the number of distinct ways to partition $2 m$ points using concepts from $\mathcal{C}$ in $\boldsymbol{R}^{k}$. Adding up over all the hypotheses, we get that

$$
\operatorname{Pr}(B) \leq C(2 m, k) e^{-\epsilon m / 32} .
$$

For the value of $m$ considered in the theorem, we have $\operatorname{Pr}(B)<\delta / 2$ and hence $\operatorname{Pr}(A)<\delta$ as required.

\section{References}

Achlioptas, D. (2001). Database friendly random projections. In Proc. Principles of Database Systems (PODS) (pp. 274-281).

Agmon, S. (1954). The relaxation method for linear inequalities. Canadian Journal of Mathematics, 6(3), 382-392.

Arriaga, R. I., \& Vempala, S. (1999). An algorithmic theory of Learning: Robust concepts and random projection. In Proc. of the 39th IEEE Foundations of Computer Science.

Balcan, N., Blum, A., \& Vempala, S. (2004). On kernels, margins and low-dimensional mappings. In Proc. of Algorithmic Learning Theory.

Bartlett, P., \& Shawe-Taylor, J. (1998). Generalization performance of support vector machines and other pattern classifiers, In B., Schvlkopf, C., Burges, \& A.J. Smola, (eds.), Advances in kernel methodssupport vector learning. MIT press.

Baum, E. B. (1990). On learning a union of half spaces. journal of Complexity, 6(1), 67-101.

Ben-David, S., Eiron, N., \& Simon, H.(2004). Limitations of learning via embeddings in euclidean half spaces. Journal of Machine Learning Research, 3, 441-461.

Blum, A., Frieze, A. Kannan, R., \& Vempala, S. (1996). A polynomial-time algorithm for learning noisy linear threshold functions. In Proc. of the 37th IEEE Foundations of Computer Science.

Blum, A., \& Kannan, R. (1993). Learning an intersection of $k$ halfspaces over a uniform distribution. In Proc. of the 34th IEEE Symposium on the Foundations of Computer Science.

Blumer, A., Ehrenfeucht, A., Haussler, D., \& Warmuth, M. K. (1989). Learnability and the vapnik-chervonenkis dimension. Journal of ACM, 36(4), 929-965.

Bylander, T. (1994). Learning linear threshold functions in the presence of classification noise. In Proc 7th Workshop on Computational Learning Theory.

Cohen, E. (1997). Learning noisy perceptrons by a perceptron in polynomial time. In Proc. of the 38th IEEE Foundations of Computer Science.

Cortes, C., \& Vapnik, V. (1995). Support-vector networks. Machine Learning, 20, 273-297.

Dasgupta, S., \& Gupta, A. (1999). An elementary proof of the Johnson-Lindenstrauss Lemma. Tech Rep. U.C. Berkeley.

Feller, W. (1957). An introduction to probability theory and its applications. John Wiley and Sons, Inc.

Frankl, P., \& Maehara, H. (1988). The Johnson-Lindenstrauss Lemma and the Sphericity of some graphs, $J$ Comb. Theory, B 44, 355-362.

Freund, Y., \& Schapire, R. E. (1999). "Large margin classification using the perceptron algorithm. Machine learning, 37(3), 277-296. 
Garg, A., Har-Peled, S., \& Roth, D. (2002). On generalization bounds, projection profile, and margin distribution. ICML, 171-178.

Garg, A., \& Roth, D. (2003). Margin distribution and learning. ICML, 210-217.

Glass, A.L., Holyoak, K.J., \& Santa J.L. (1979). The structure of categories. In Cognition. Addison-Wesley.

Grötschel, M., Lovász, L., \& Schrijver, A. (1988). Geometric algorithms and combinatorial optimization, Springer.

Hoeffding, W. (1963). Probability inequalities for sums of bounded random variables. Journal of the American Statistical Association, 58, 13-30.

Indyk, P., \& Motwani, R. (1998). Approximate nearest neighbors: Towards removing the curse of dimensionality. In Procedings of ACM STOC.

Johnson, W. B., \& Lindenstrauss, J.(1984). Extensions of lipshitz mapping into Hilbert space. Contemporary Mathematics, 26, 189-206.

Kearns, M.J., \& Schapire, R.E. (1994). Efficient distribution-free learning of probabilistic concepts. Journal of Computer and System Sciences, 48(3), 464-497.

Kearns, M.J., \& Vazirani, U. (1994). Introduction to computational learning theory. MIT Press.

Kleinberg, J. (1997). Two algorithms for nearest-neighbor search in high dimensions. In Procedings 29th ACM Symposium on Theory of Computing.

Klivans, A., \& Servedio, R. (2004). Learning intersections of halfspaces with a margin, In Procedings 17th Workshop on Computational Learning Theory.

Knowlton, B. (1999). What can neuropsychology tell us about category learning. Trends in Cognitive Science, 3, 123-124.

Komatsu, L.K. (1992). Recent views on conceptual structure. Psychological Bulletin, 112(3).

Linial, N., London, E., \& Rabinovich, Y. (1994). The geometry of graphs and some of its algorithmic applications. In Proc. of 35th IEEE Foundations of Computer Science.

Littlestone, N. (1987). Learning quickly when irrelevant attributes abound: A new linear-threshold algorithm. Machine Learning, 2,285-318.

Littlestone, N. (1991). Redundant noisy attributes, attribute errors, and linear threshold learning using winnow. In Proc. 4th workshop on computational learning theory.

Mandler, J. M. (2003). Conceptual Categorization, Chapter 5. In D. H., Rakinson, \& L. M. Oakes, (eds.), Early category and concept development: Making sense of the blooming, buzzing confusion. Oxford University Press.

Minsky, M., \& Papert., S., (1969). Perceptrons: An introduction to computational geometry. The MIT press.

Nosofsky, R., \& Zaki, S., (1969). Math modeling, neuropsychology, and category learning: Response to B. Knowlton (1999). Trends in Cognitive Science, 3, 125-126, 1999. Perceptrons: An introduction to computational geometry. The MIT press.

Rakinson, D. H., \& Oakes, L. M. (eds.), (2003). Early category and concept development: Making sense of the blooming, buzzing confusion. Oxford University Press.

Reed, S. K. (1982). Categorization, in cognition: Theory and applications. brooks/cole.

Reed, S. K., \& Friedman, M. P. (1973). Perceptual vs. conceptual categorization. Memory and Cognition, 1.

Rosch, E. (1978). Principles of categorization. in E., Rosch, \& Lloyd, B. B. (eds.), Cognition and categorization Hillsdale.

Rosch, E. H., Mervis, C. B., Gray, W. D., Johnson, D. M., \& Boyes-Braem, P. (1976). Basic ojects in natural categories. Cognitive Psychology, 8.

Rosenblatt, F. (1962). Principles of neurodynamics. Spartan Books.

Schapire, R. E., Freund, Y., Bartlett, P. L., \& Lee, W. S. (1998). Boosting the margin: A new explanation for the effectiveness of voting methods. Annals of Statistics, 26(5), 1651-1686.

Valiant, L. G. (1984). A theory of the learnable. Communications of the ACM, 27(11), 1134-1142.

Valiant, L. G. (1998). A neuroidal architecture for cognitive computation. In Proc. of ICALP.

Vapnik, V. N. (1995). The nature of statistical learning theory. Springer.

Vapnik, V. N., \& Chervonenkis, A. Ya., (1971). On the uniform convergence of relative frequencies of events to their probabilities. Theory of Probability and its applications, 26(2), 264-280.

Vempala, S. (1997). A random sampling based algorithm for learning the Intersection of Half-spaces. In Proc. of the 38th IEEE Foundations of Computer Science.

Vempala, S. (2004). The random projection method. 65. DIMACS series, AMS. 